\title{
Designing and Researching about A New Type of Mechanical-Electronic Percussion Equipment
}

\author{
Yong Xiao ${ }^{1, \text { a }}$ Fei Ning ${ }^{2, b}$, Yuqin Wen ${ }^{2, c}$ and Kai Sang ${ }^{2, d}$ \\ ${ }^{1,2}$ Beijing Shengfeifan Electronic System Technology Development CO.LTD, Beijing 102209, China. \\ awyq_bupt@163.com
}

Keywords: Mechanical percussion, electrical percussion, electromagnet, firing pin, ANSYS Workbench.

\begin{abstract}
During the disassemble of dangerous ammunition, there are always electronic primers and mechanical primers that cannot be dismantled. Based on these occasions, we design a new equipment which can fire both electronic and mechanical primers. This equipment saves the ammunition disassemble cost a lot.
\end{abstract}

\section{Introduction}

When disassembly the dangerous ammunition, there are always electronic primers and mechanical primers that cannot be disassembled easily. On this occasion, primer firing equipments are needed to fire the primers and disassembly the dangerous ammunition[1]. Based on the design, we do the simulation work with the help of ANSYS Workbench 15.0[2].

\section{Structure Design}

This new type of primer firing equipment consists four parts, includes firing mechanism, clamping mechanism, DC power and sink. Which design a Mechanical-Electronic firming mode innovatively, makes the percussion process more stable and reliable.

Clamping mechanism and firing mechanism was installed on the fire platform. Firing mechanism includes the compaction structure and supporting structure. There is a groove near the supporting structure, which can limit the degree of freedom on the horizontal direction.

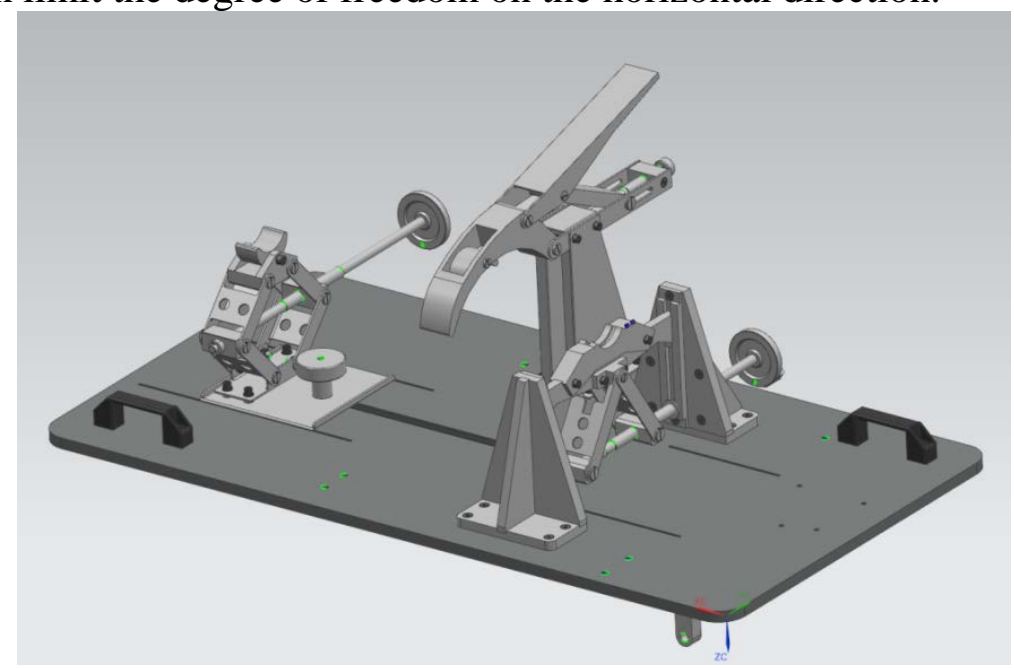

Fig. 1 The fire platform

Firing mechanism includes electromagnet holder, electromagnet, firing pin, reset spring. Firing mechanism can move on the horizontal direction to adjust the distance between the different percussion and firing pin to make the force reach the highest value. The moment the electromagnet switching on, the firing pin stretches out to fire the percussion. This force can reach $350 \mathrm{~N}$, far outweigh the $200 \mathrm{~N}$ needed force value, make the equipment more liable. Besides, it can make sure the accomplishment of every percussion successfully, and avoid the dangerous accident because of the lack of the force. 
Clamping mechanism includes a pair of supporting structure and the compaction structure. The supporting structure can be adjusted by a joystick to make sure the concentric of the percussion and firing pin, which can adjust different diameter percussion's firing work. When the height of the supporting structure is adjusted, lay the cartridge on it, then put the compaction structure on the cartridge, make sure the cartridge is fixed on the platform.

DC power is set inside the box which is under the platform. two power sources are linked in series, to provide stable current and voltage.

Sink is made separated with the DC power box. When the primer is firing, fill the sink with water, tilt the platform to make the cartridge immerge under the water, so the primer is firing to the water, make the noise and pollution less and achieve green elimination.

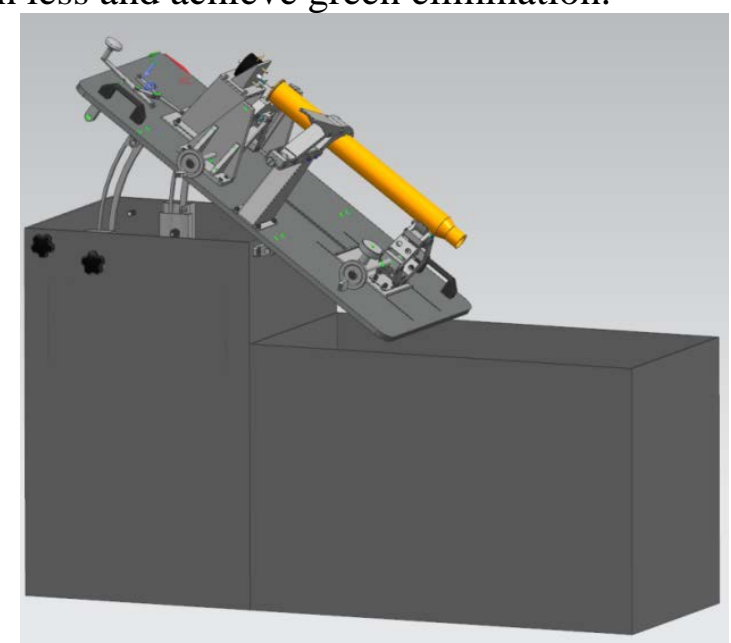

Fig. 2 Fire the primer to the water

\section{Theory of the percussion}

This new type of percussion firing equipment can accomplish two firing modes, mechanical firing and electronic firing. Firing mechanism includes electromagnet holder, electromagnet, firing pin, reset spring, mode choosing switch, secure switch, firing switch.

Firing theory is showed as the following figure. Mode choosing switch is used to choose firing mode; secure switch is used to fire the percussion automatically when the cartridge is compacted safely; firing switch is used as a switch that operation staff can operate it. During these structures, firing switch links to the ground when not working.

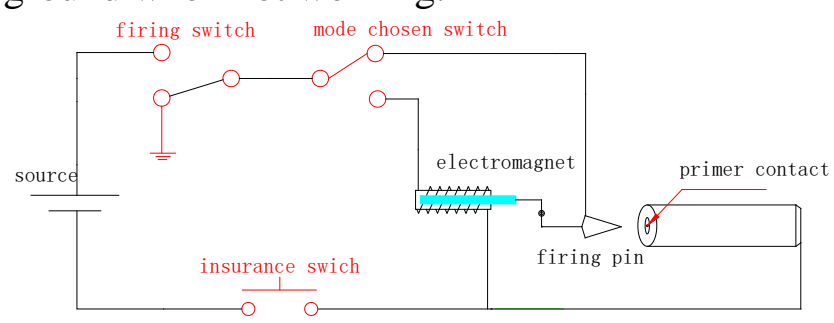

Fig. 3 Firing theory

Put the mode choosing switch to mechanical firing mode, push the firing switch, the electromagnet electrify, firing pin comes out, the electronic-percussion is firing to the water. Reset spring make the electromagnet back, to prepare the next firing work.

Put the mode choosing switch to electronic firing mode, push the firing switch, the electromagnet electrify, make a contact between firing pin and electronic-percussion, the circuitry become circle, make the percussion firing eventually. Reset spring make the electromagnet back, to prepare the next firing work. 


\section{Mechanical Analysis}

The electromagnet is push-pull type, mainly consists shell, fixed iron core, mobile iron core and loop. When the loop set up an electric circuit, mobile iron core moves in the horizontal direction so that it can push or pull the external load. because of the using of high-magnetic material and cone-magnetic-loop structure, the electromagnet is high-quality than the usual tiny magnetic in displacement and suction. Based on the actual situation of the whole system, an electromagnet is chosen TS7010 which tour is $0 \sim 12 \mathrm{~mm}$ and the highest suction of it is $350 \mathrm{~N}$, far more effective than the $200 \mathrm{~N}$ in theory[3]. Checking the displacement-suction curve of TS7010 electromagnet, we can tell that when the displacement is $10 \mathrm{~mm}$, the suction is highest.

Measure relevant sizes of TS7010. Length $\mathrm{L}_{1}=80 \mathrm{~mm}, \mathrm{~L}_{2}=12 \mathrm{~mm}, \mathrm{~L}_{3}=15 \mathrm{~mm}$, diameter $\mathrm{D}_{1}=8 \mathrm{~mm}, \mathrm{D}_{2}=15 \mathrm{~mm}, \mathrm{D}_{3}=5 \mathrm{~mm}, \mathrm{D}_{1}=8 \mathrm{~mm}, \mathrm{D}_{2}=15 \mathrm{~mm}, \mathrm{D}_{3}=5 \mathrm{~mm}$.

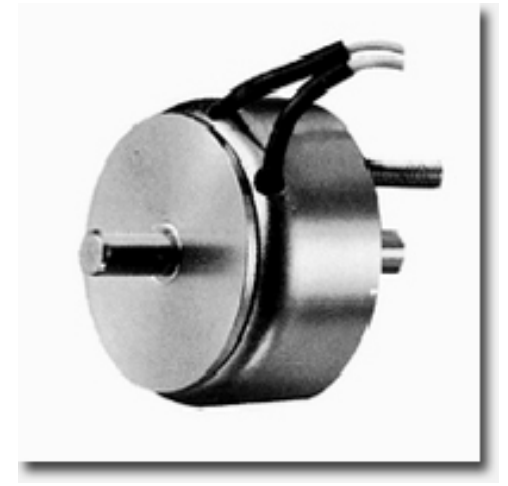

Fig. 4 TS7010 electromagnet

Make the displacement of the firing pin is s, the force is F, firing pin's mass is $\mathrm{M}$, the area of the contact between firing pin and cartridge is A. The moment the firing pin hit the cartridge, the stress of the contact is:

$$
\mathrm{E}=\frac{\mathrm{F}}{\mathrm{A}} \text {. }
$$

Compare $\mathrm{E}$ and the yield stress of the primer, $\mathrm{E}>\mathrm{e}$, then the primer is firing successfully.

\section{Finite Element Modeling and Simulating Analysis}

Take one type of antiaircraft gun primer for an instance.

Simulation environment is ANSYS Workbench 15.0. Assuming an ideal situation, firing pin isn't dismiss the primer after the hit, and there is no friction and spring back[4].

Set up the material character, firing pin and primer's parameters are as follows.

Table 1 Parameters of firing pin and primer's

\begin{tabular}{|c|c|c|}
\hline Category & Firing pin & Primer \\
\hline Density $\left(\mathrm{kg} / \mathrm{m}^{3}\right)$ & 7900 & 8930 \\
\hline Elastic modulus(GPa) & 206 & 97 \\
\hline Poisson ratio & 0.27 & 0.42 \\
\hline Shear modulus(GPa) & 81 & 34 \\
\hline
\end{tabular}

Among these parameters, shear modulus is generated automatically when other parameters are set in the software. Generate grid Mesh result is as following figure.

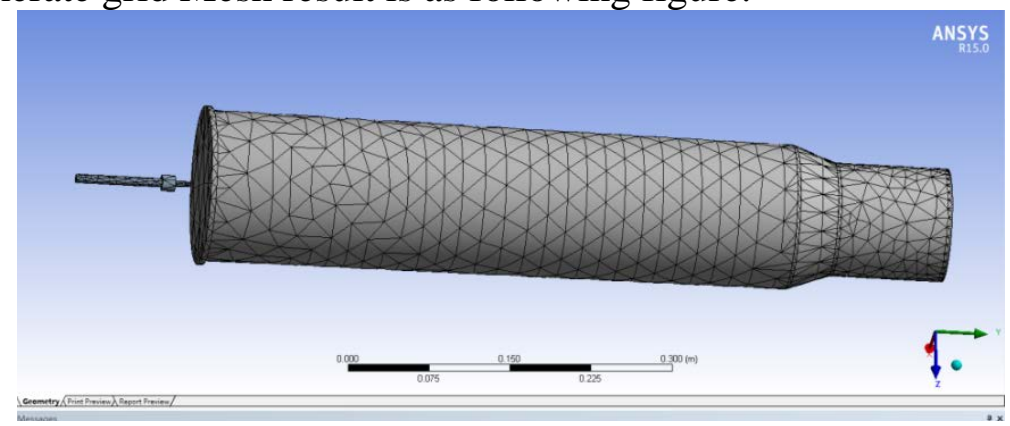

Fig. 5 Meshing result 
Bring 350N's force to cartridge's back surface, the stress, strain and total deformation's cloud picture showed as follows.

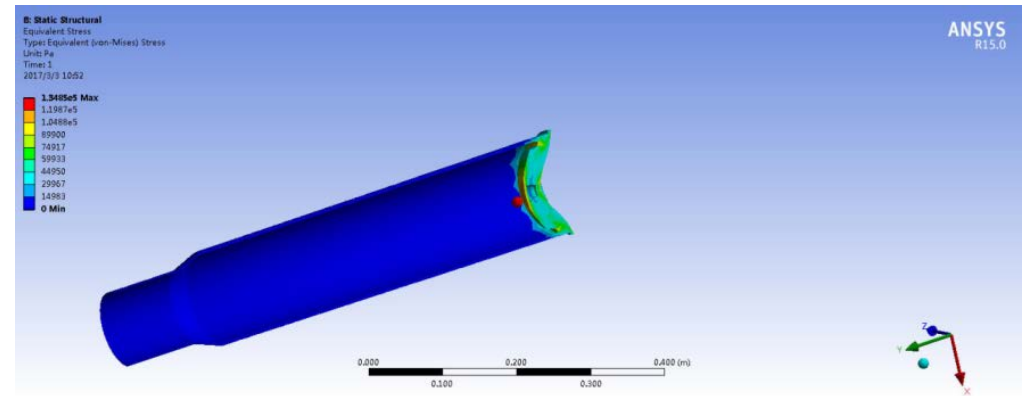

Fig. 6 Stress cloud picture

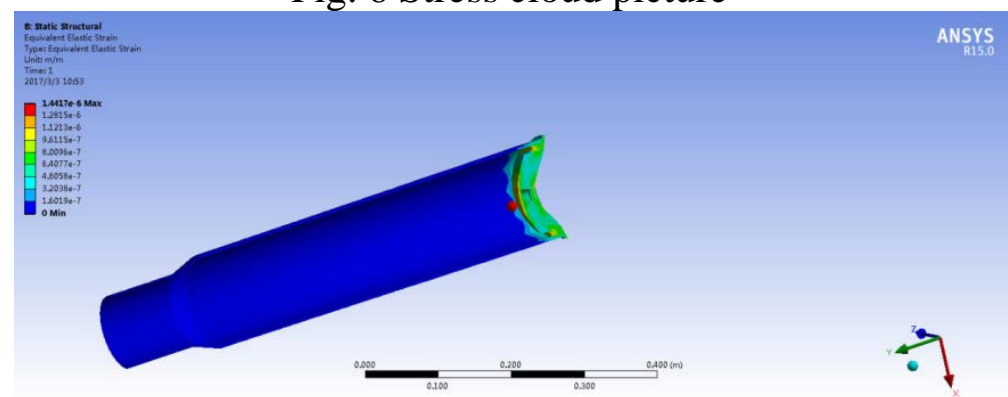

Fig. 7 Stain cloud picture

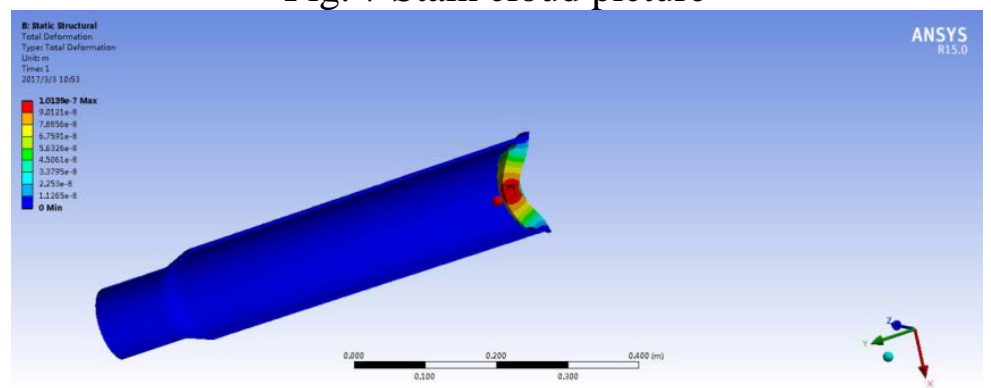

Fig. 8 Total deformation cloud picture

From the cloud picture, we can tell 350N's force is definitely enough for the firing work.

\section{Summary}

This article introduces a new equipment which can fire both electronic and mechanical primers. Compared with the traditional equipment, it has a great advantage. The operation procedures are simple and it realize green elimination. With the help of ANSYS Workbench software, we simulate the firing moment, which proves the equipment can fire the primer safety and efficiency. This new equipment solve a problem in primer firing area.

\section{References}

[1]. Zhao Junli, Pan Yutian, Li Peiying. Invalidity Analysis and Improved Measures for Eletromechanic Percussion Device[J]. Journal of Gun Launch and Control,2004 (2): 40-43.

[2]. Jing Yinping, Yang Zhen. Application of Finite Element Method in Percussion of Firearms[J].Mechanical Engineering \& Automation,2007 3(142): 48-52.

[3]. Huo Jianpeng, Gao Ran, Pan Yutian. Impact Mechanics Analysis of Mechanical Percussion Device[J]. Mechanical Engineering \& Automation,2011 1(164): 82-84.

[4]. Wang Hongmei, Yang Wei,Liu Jun. Simulation Study on collisional Energy Loss in Firing Mechanism[J].Computer Simulation,20013 30(2): 51-54. 